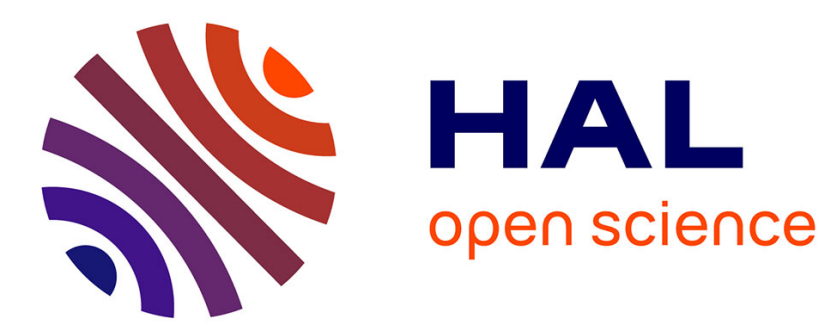

\title{
Locking of Intrinsic Angular Momenta in the Capture of Quadrupole Diatoms by Ions
}

\author{
Juergen Troe, Evgueni E. Nikitin, E. I. Dashevskaya, I. Litvin
}

\section{To cite this version:}

Juergen Troe, Evgueni E. Nikitin, E. I. Dashevskaya, I. Litvin. Locking of Intrinsic Angular Momenta in the Capture of Quadrupole Diatoms by Ions. Molecular Physics, 2010, 108 (07-09), pp.873-882. 10.1080/00268970903501717 . hal-00596271

\section{HAL Id: hal-00596271 \\ https://hal.science/hal-00596271}

Submitted on 27 May 2011

HAL is a multi-disciplinary open access archive for the deposit and dissemination of scientific research documents, whether they are published or not. The documents may come from teaching and research institutions in France or abroad, or from public or private research centers.
L'archive ouverte pluridisciplinaire HAL, est destinée au dépôt et à la diffusion de documents scientifiques de niveau recherche, publiés ou non, émanant des établissements d'enseignement et de recherche français ou étrangers, des laboratoires publics ou privés. 


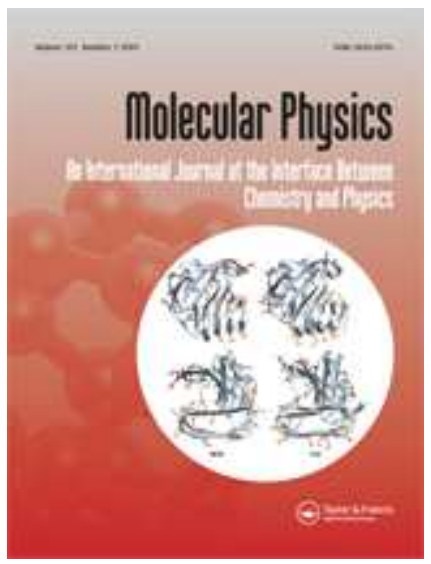

\section{Locking of Intrinsic Angular Momenta in the Capture of Quadrupole Diatoms by Ions}

\begin{tabular}{|r|l|}
\hline Journal: & Molecular Physics \\
\hline Manuscript ID: & TMPH-2009-0326 \\
\hline Manuscript Type: & Special Issue Paper - In honour of Prof Richard Zare \\
\hline Author: & 22-Oct-2009 \\
\hline Complete List of Authors: & $\begin{array}{l}\text { Troe, Juergen; Universitaet Goettingen, Institut für Physikalische } \\
\text { Chemie } \\
\text { Nikitin, Evgueni; Technion-Israel Institute of Technology } \\
\text { Dashevskaya, E.; Technion-Israel Institute of Technology } \\
\text { Litvin, I.; Technion-Israel Institute of Technology }\end{array}$ \\
\hline Keywords: & $\begin{array}{l}\text { Locking of angular momenta, Capture processes, Adiabatic channel } \\
\text { model }\end{array}$ \\
\hline \\
\hline $\begin{array}{l}\text { Note: The following files were submitted by the author for peer review, but cannot be converted } \\
\text { to PDF. You must view these files (e.g. movies) online. }\end{array}$ \\
\hline 0326.zip \\
\hline
\end{tabular}

\section{s ScholarONE \\ Manuscript Central}




\title{
Locking of Intrinsic Angular Momenta in the Capture of Quadrupole Diatoms by Ions
}

\author{
E. I. Dashevskaya ${ }^{a, b}$, I. Litvin ${ }^{a, b}$, E. E. Nikitin ${ }^{a, b}$, and J. Troe ${ }^{b, c, *}$ \\ ${ }^{a}$ Schulich Faculty of Chemistry, Technion - Israel Institute of Technology \\ Haifa, 32000, Israel \\ ${ }^{b}$ Max-Planck-Institut für Biophysikalische Chemie, \\ Am Fassberg 11, Göttingen D-37077, Germany \\ ${ }^{c}$ Institut für Physikalische Chemie, Universität Göttingen, \\ Tammannstrasse 6, Göttingen D-37077, Germany \\ *Email: shoff@gwdg.de
}

\begin{abstract}
The capture dynamics of rotationally polarized homonuclear diatoms in lowenergy collisions with ions is studied theoretically. The interaction potential includes charge-quadrupole and charge-induced dipole terms. The former, taken in the perturbed rotor approximation, exhibits an $R^{-3}$ anisotropic dependence and causes an only gradual locking of the intrinsic angular momentum of the diatom to the collision axis. As a result, the capture occurs in a regime of incomplete locking, and the passage over centrifugal barriers is accompanied by considerable gyroscopic effects. The $j$-specific capture cross sections for rotationally-aligned diatoms show a marked dependence on the polarization state which is not described by the conventional adiabatic channel model. The latter, however, provides a fair approximation for the cross sections of unpolarized diatoms. These features are due to a considerable angle of rotation of the collision axis before the barrier is reached and to a small angle of rotation during the passage across the barrier. The numerically determined, scaled, capture cross sections are represented by an approximate analytical expression that interpolates between two limiting cases, very small (adiabatic channel model) and very large (fly wheel model) gyroscopic effects of the rotating diatom.
\end{abstract}




\section{Introduction}

In 1982, Rettner and Zare [1] introduced the notion of "orbital following" in the context of reactive collisions and discussed, in qualitative terms, the sudden and adiabatic regimes of the "orbital following". A similar phenomenon was known earlier for the field of atomic collisions (see e.g. the review [2]). For instance, Dashevskaya and Mokhova [3], already in 1973, have used the angular momentum recoupling in the calculation of depolarization cross sections of atomic collisions. They assumed a sudden transformation of atomic functions into molecular ones at a certain interatomic separation called the "matching radius". The model of the "orbital following" and the "sudden matching" was generalized later in a number of articles (see the reviews [4,5] and the book [6]) where the concept of the locking radius $R_{L}$ was introduced and the non-adiabatic behavior of the angular momentum states within the recoupling region of extension $\delta R_{L}$ was discussed. In particular, the case of an atom in a state $j=1$ was treated in detail [7] and a classical model was elaborated for $j>>1$ [8]. It appeared expedient to consider the locking in a framework of successions, when atoms approach or recede, of the Hund coupling cases $a, b, c, d, e$ in a diatom $[9,10]$ or the $\alpha, \beta, \gamma, \delta, \varepsilon$ coupling cases for a more complicated system, atom + diatomic molecule [11]. Basically, the locking occurs at the distance $R \approx R_{L}$ where the precession frequency of the intrinsic angular momentum about the collision axis, $\omega_{\text {prec }}(R)$, is close to the characteristic frequency of the Coriolios interaction,$\omega_{\text {Cor }}(R)$, which, in turn, is proportional to the frequency of rotation of the collision axis , $\omega_{\text {rot }}(R)$, for a given value of the total angular momentum. The range of the locking region, $\delta R_{L}$, that encompasses the interplay between the coupling of the intrinsic momentum to the collision axis (precession) and its decoupling from this axis due to the gyroscopic effect (Coriolis interaction) is proportional to the inverse logarithmic derivative of $\omega_{\text {prec }}(R)$ with respect to $R$. In short, $R_{L}$ and $\delta R_{L}$ are roughly determined by the expression

$$
\begin{gathered}
\omega_{\text {prec }}\left(R_{L}\right) \approx \omega_{\text {Cor }}\left(R_{L}\right) \\
\delta R_{L} \approx \mid d \ln \left(\omega_{\text {prec }}(R) /\left.d R\right|_{R=R_{L}}\right.
\end{gathered}
$$


The nonadiabatic interaction in a region centered at $R_{L}$ determines a part of the total scattering matrix, which can be incorporated into the latter by adiabatic matching. However, the interest for the study of the locking phenomenon as a half-collision event diminished once the locking could be handled by the powerful numerical codes for calculating full-collision or half-collision multichannel scattering matrices for atom-atom and atom-diatom systems such as discussed, e.g., in recent work on the theory of photodissociation (see refs $[12,13]$ and the work cited therein). In this way, the account for long-range Coriolis coupling between asymptotically-degenerate states of the collision partners represents a considerable step forward compared to the pure statistical description of angular momentum recoupling in the formation and decay of long-lived molecular complexes $[14,15]$. Due to the rotational coupling, new effects arise termed “dynamical polarization" [16].

On the other hand, the standard method of calculating rate coefficients and product distribution functions for processes that proceed via formation of long-lived complexes, still uses the approaches that assume the conservation of the projection of the intrinsic angular momentum of partners onto the collision axis. This is the statistical adiabatic channel (AC) model of [17], also known, in its later versions, as the perturbed rotational (PR) state method [18], the adiabatic invariance (AI) method [19], and the adiabatic capture centrifugal sudden (ACCS) approximation [20]. These models were shown to be equivalent to each other, apart from the effects of more or less adequate numerical simplifications [20-22]. Within the AC approach, the formation of the complex is associated with the passage across the barrier of the effective potential formed by superposition of the centrifugal energy and the AC potential. The latter is labeled, beside others, also by the parameter $m$. Taken literally, $m$ can be interpreted as the projection of the intrinsic angular momentum onto the collision axis at large values of $R$, i.e. the locking is assumed to occur in the asymptotic region of $R$. This is clearly not quite correct, and, therefore, the question arises about the relation between the $m$, that enter into the definition of AC potentials, and their asymptotic counterparts. The question here is answered in terms of the locking matrix, provided that the locking distance $R_{L}$ is substantially larger than the position of the barriers $R_{m}^{*}, R_{L} \gg R_{m}^{*}$, and that the extent of 
the region, $\delta R_{L}$, where the recoupling of the angular momentum occurs, is small, $\delta R_{L}<<R_{L}$. These two conditions are fulfilled at worst when the interfragment interaction is of long range, and, therefore, this case deserves detailed study without separating the capture event into the barrier crossing at a fixed value of $m$, and recoupling of the angular momentum far before the barrier is reached. It appears therefore interesting to consider an anisotropic potential proportional to $R^{-3}$ which is the leading long-range interaction for which the locking is possible in principle. A physical object exhibiting this type of interaction is the charge-quadrupolar rotor system. (Note that for an anisotropic potential proportional to $R^{-2}$ which is characteristic for a charge-dipolar rotor system, the locking does not occur at all since the potential and the Coriolis coupling show the same $R$-dependence, and Eq (1) has no solution at finite $R$ ).

In the following article we consider the capture of a quadrupolar rotationallypolarized diatom in collisions with an ion in the regime when the perfect locking of the angular momentum to the collision axis in the region of centrifugal barriers is questionable. We adopt the perturbed rotor approximation for the Hamiltonian of this system and the classical description of the locking and capture dynamics. The first constraint is consistent with our earlier observations that the anisotropic quadrupolar interaction shows up on the background of isotropic charge-induced dipole interactions at low collision energies (applicability of the PR approximation). The second one is in line with our previous work on the study of classical capture [23, 24]. We thus calculate the capture probability as a function of total angular momentum, intrinsic angular momentum and its projection onto the relative velocity vector, collision energy and the parameters of charge-quadrupole and charge-induced dipole interactions. In addition we determine the respective capture cross sections for axially-symmetric polarized diatoms. In this way we generalize our earlier work on capture in translational-rotational canonical ensembles [23] and in $j$-specific translational canonical ensembles [24] of unpolarized collision partners. This also provides more detailed insight into the capture dynamics of polarized diatoms compared to earlier work [25]. The plan of the article is the following. In Section 2 we specify the classical Hamiltonian that describes capture beyond the AC approximation. In Section 3 we discuss the locking/capture dynamics in terms of the 
properties of some characteristic trajectories. Section 4 presents the results of calculating capture cross sections for axially-aligned diatoms. Section 5 concludes the article.

\section{Perturbed rotor Hamiltonian for an ion-diatomic rigid rotor system.}

The interaction of a homonuclear diatom with a charged particle includes chargequadrupole and charge-induced dipole interaction terms

$U(R, \gamma)=\frac{q Q}{R^{3}} P_{2}(\cos \gamma)-\frac{q^{2} \alpha}{2 R^{4}}$

where $R$ is the charge-diatom separation, $\gamma$ is the angle between molecular the axis $\mathbf{n}$ and the collision axis $\mathbf{R}, P_{2}(\cos \gamma)$ is the Legendre polynomial, and $q, Q, \alpha$ are the charge of the particle, the quadrupole moment and polarizability of the diatom, respectively. The potential in the perturbed rotor approximation, $U^{P R}$, is derived from Eq (2) by averaging $U$ over the angle of proper rotation of the rotor with the fixed angle $\beta$ between vectors $\mathbf{j}$ and $\mathbf{R}$. This is accomplished by using the addition theorem for Legendre polynomials (see e.g. [26]) with subsequent averaging over appropriate azimuthal angle, as discussed in $[14,15]$. The application of this approach to $P_{2}(\cos \gamma)$ yields $-P_{2}(\cos \beta) / 2$ such that Eq (2) transforms into

$$
U^{P R}(R, \beta)=\frac{-q Q}{2 R^{3}} P_{2}(\cos \beta)-\frac{q^{2} \alpha}{2 R^{4}}
$$

The PR Hamiltonian is constructed as a sum of the relative kinetic energy and the PR potential energy:

$$
H^{P R}=\frac{P_{R}^{2}}{2 \mu}+\frac{L^{2}}{2 \mu R^{2}}+U^{P R}(R, \beta)
$$


where $\mu$ is the reduced mass of the collision partners, $P_{R}$ is the momentum conjugate to $R$ and $L^{2}$ is the square of the relative angular momentum. Once it is expressed in canonical variables, the Hamiltonian in Eq (4) generates equations of motion. These equations assume a simple form if the conservation of the total angular momentum $\mathbf{J}$ is explicitly taken into account.

If one chooses a body-fixed frame, one replaces $L^{2}$ by $(\mathbf{J}-\mathbf{j})^{2}$ and introduces the dynamical variables $\phi$, the azimuthal angle of $\mathbf{j}$ in the plane normal to the collision axis, and $P_{\phi}$, the projection of the intrinsic angular momentum onto the collision axis. On the other hand, if one chooses a spaced-fixed frame and the vector $\mathbf{J}$ as a polar axis, one expresses $L^{2}$ through polar and azimuthal angles of the collision axis, $\theta, \varphi$, and conjugate momenta $P_{\theta}, P_{\varphi}$; in addition, $P_{2}(\cos \beta)$ should be expressed as a certain function of $\theta, \varphi$ and of two other angular variables that fix the orientation of the vector $\mathbf{j}$ in the SF frame. This can be done also via addition the theorem of the Legendre polynomials. In the following, we adopt the BF frame, and use the SF frame only for discussion of certain approximations (see below).

The perturbed rotor Hamiltonian $H^{P R}$ in the BF frame contains two dynamical degrees of freedom (the radial relative motion of the partners and the precessional motion of $\mathbf{j}$ about $\mathbf{R}$ ); beside $q, Q, \alpha$, it contains two other parameters, the conserved scalar quantities $J$ and $j$. It is expedient to pass to scaled variables and parameters, and replace the PR Hamiltonian $H^{\mathrm{PR}}$ by a scaled Hamiltonian $\eta^{\mathrm{PR}}$ recognizing the conservation of the total energy $E$. The scaling is achieved by the transformations

$$
\begin{array}{ll}
\rho=\left(E^{1 / 3}|q Q|^{-1 / 3}\right) \times R, & \pi_{\rho}=\left(E^{-1 / 2} \mu^{-1 / 2}\right) \times P_{R} ; \\
\pi_{\phi}=\left(E^{-1 / 6} \mu^{-1 / 2}|q Q|^{-1 / 3}\right) \times P_{\phi} ; & \tau=\left(E^{5 / 6} \mu^{-1 / 2}|q Q|^{-1 / 3}\right) \times t \\
(\mathrm{I}, \mathrm{\imath})=\left(E^{-1 / 6} \mu^{-1 / 2}|q Q|^{-1 / 3}\right) \times(J, j) ; & \lambda=\alpha\left|q / Q^{2}\right|^{2 / 3} E^{1 / 3}
\end{array}
$$

The scaled PR Hamiltonian $\eta^{\mathrm{PR}}$ is defined as $\eta^{\mathrm{PR}}=H^{\mathrm{PR}} / E$ such that the dynamics in reduced variables corresponds to the scaled total energy equal to unity. From now on, we 
drop superscript PR since we adhere to this approximation throughout this work. Then, the explicit form of the Hamiltonian $\eta_{s} \equiv \eta_{s}\left(\rho, \pi_{\rho}, \phi, \pi_{\phi} ; \mathrm{I}, 1, \lambda\right)$, (with the semicolon separating dynamical variables and parameters), reads

$$
\eta_{s}\left(\rho, \pi_{\rho}, \phi, \pi_{\phi} ; \mathrm{I}, \mathrm{l}, \lambda\right)=\frac{\pi_{\rho}^{2}}{2}+v_{\text {eff,s }}\left(\rho, \phi, \pi_{\phi} ; \mathrm{I}, \mathrm{l}, \lambda\right)
$$

and

$$
\begin{aligned}
& \mathrm{U}_{\text {eff }, s}\left(\rho, \phi, \pi_{\phi} ; \mathrm{I}, \mathrm{\imath}, \lambda\right)= \\
& =\frac{\mathrm{I}^{2}+\mathrm{\imath}^{2}-2 \pi_{\phi}^{2}-2 \sqrt{\mathrm{I}^{2}-\pi_{\phi}^{2}} \sqrt{\mathrm{l}^{2}-\pi_{\phi}^{2}} \cos \phi}{2 \rho^{2}}-\frac{s}{2 \rho^{3}} P_{2}\left(\pi_{\phi} / \imath\right)-\frac{\lambda}{2 \rho^{4}}
\end{aligned}
$$

where the superscript $s=\operatorname{sign}(q Q)$ appears as a result of the scaling through the absolute value of the product $q Q$. The first term at the r.h.s. of Eq (6) is the radial kinetic energy and the second can be regarded as an effective potential energy for the radial motion. The latter, given by Eq (7), includes the centrifugal energy with the Coriolis interaction, the ACPR counterpart of the charge-quadrupole interaction, and the charge-induced dipole interaction. The Hamiltonian $\eta_{s}$ generates the canonical equations of motion for the evolution of the four quantities $\rho(\tau), \pi_{\rho}(\tau), \phi(\tau), \pi_{\phi}(\tau)$ subject to certain initial conditions. In this work, we assumed a uniform distribution over the initial azimuthal angle $\phi_{i}$ and have taken $\rho_{i}$ large enough to set $\pi_{\rho, i}=\sqrt{2}$ that corresponds to the initial scaled energy $\varepsilon$ equal to unity. Then the only essential parameter is the initial alignment index $\zeta_{i}=\pi_{\phi i} / l$. The trajectories were terminated either when $\rho(\tau)$ become again large after reflection from the barrier (reflected trajectory) or very small (capture trajectory), after passing through the barrier. In order to monitor this behavior, we have calculated also the time dependence of the effective potential $v_{\text {eff }}(\tau)$ along the trajectories. 
Two approximate Hamiltonians can be derived from the general PR Hamiltonian, that correspond to the conditions when the intrinsic angular momentum $j$ is substantially smaller or larger than a typical angular momentum $\bar{L}$ corresponding to the capture

For $j<<\bar{L}$, one also has $1<<\mathrm{I}$. Neglecting in Eq (7) the two last terms in the expression for the centrifugal energy, one gets the standard expression for the ACPR Hamiltonian:

$$
\begin{aligned}
& \eta_{s}^{\mathrm{AC}}\left(\rho, \pi_{\rho} ; \mathrm{I}, \varsigma^{\mathrm{AC}}, \lambda\right)=\frac{\pi_{\rho}^{2}}{2}+v_{\mathrm{eff}, s}^{\mathrm{AC}}\left(\rho ; \mathrm{I}, \varsigma^{\mathrm{AC}}, \lambda\right) \\
& \mathrm{v}_{\mathrm{eff}, s}^{\mathrm{AC}}\left(\rho ; \mathrm{I}, \varsigma^{\mathrm{AC}}, \lambda\right)=\frac{\mathrm{I}^{2}}{2 \rho^{2}}-\frac{s}{2 \rho^{3}} P_{2}\left(\varsigma^{\mathrm{AC}}\right)-\frac{\lambda}{2 \rho^{4}}
\end{aligned}
$$

in which the constant alignment index $\varsigma^{\mathrm{AC}}$ appears as a consequence of the conservation of the dynamical variable $\pi_{\phi}=\omega$, i.e. $\varsigma^{\mathrm{AC}}=\omega / \mathrm{l}$. The maximum of $v_{\mathrm{eff}, s}^{\mathrm{AC}}\left(\rho ; \varsigma^{\mathrm{AC}}, \mathrm{I}, \lambda\right)$ with respect to $\rho, v_{\text {eff, } s}^{\mathrm{AC}, \mathrm{max}}\left(\varsigma^{\mathrm{AC}}, \mathrm{I}, \lambda\right)$, determines the "allowed" values of $\varsigma^{\mathrm{AC}} \equiv \varsigma_{s}^{\mathrm{AC}}$, for which the capture channels are open for given values of $\mathrm{I}, \lambda, v_{\mathrm{eff}, s}^{\mathrm{PRAC}, *}\left(\varsigma^{\mathrm{AC}}, \mathrm{I}, \lambda\right) \leq 1$. Figure 1 shows the allowed regions of $\zeta_{s}^{\mathrm{AC}}$ for $s=1$ and $s=-1$ with $\mathrm{I}=0$ bounded by the curves $\left.\varsigma_{+}^{\mathrm{AC}, \min }(\lambda) \equiv \varsigma_{s}^{\mathrm{AC}, \min }(\lambda)\right|_{s=1}$ and $\left.\varsigma_{-}^{\mathrm{AC}, \max }(\lambda) \equiv \varsigma_{s}^{\mathrm{AC}, \max }(\lambda)\right|_{s=-1}$

For $j \gg>\bar{L}$, all the terms in the numerator of the expression for the centrifugal energy are of the same order, and it is easier to derive the respective approximate Hamiltonian in the SF frame. The approximation uses the fact that since $\mathbf{j}$ is close to $\mathbf{J}$, the orientation of $\mathbf{j}$ is approximately conserved in the space (this approximation combines the sudden limit with respect to the orientation of $\mathbf{j}$ and the adiabatic limit with respect to the change of the absolute value of $\mathbf{j}$; it was called in [25] the fly-wheel, FW, approximation) and, therefore, the angle $\beta$ between $\mathbf{R}$ and $\mathbf{j}$ is approximately equal to the angle $\theta$ between $\mathbf{R}$ and $\mathbf{J}$. Identifying $\beta$ with $\theta$ in $U^{P R}$, we get the FW Hamiltonian in the form: 


$$
\begin{aligned}
& \eta_{\mathrm{s}}^{\mathrm{FW}}\left(\pi_{\rho}, \rho, \pi_{\theta}, \theta ; \mathrm{I}, \varpi\right)=\frac{\pi_{\rho}^{2}}{2}+v_{\text {eff }, s}^{\mathrm{FW}}\left(\rho, \pi_{\theta}, \theta ; \mathrm{I}, \varpi, \lambda\right) \\
& v_{\mathrm{eff}, s}^{\mathrm{FW}}\left(\rho, \pi_{\theta}, \theta ; \mathrm{I}, \varpi, \lambda\right)=\frac{1}{2 \rho^{2}}\left(\pi_{\theta}^{2}+\frac{\varpi^{2}}{\sin ^{2} \theta}\right)-\frac{s}{2 \rho^{3}} P_{2}(\cos \theta)-\frac{\lambda}{2 \rho^{4}}
\end{aligned}
$$

in which the constant projection of the relative angular momentum $\varpi$ onto the total angular momentum appears as a consequence of the cylindrical symmetry of the interaction, i.e. $\pi_{\varphi}=\varpi$. The approximate Hamiltonian in Eq (9) looks more complicated than the approximate Hamiltonian in Eq (8) because the former contains more information (motion in space) than the latter (only radial motion).

\section{Dynamics of locking}

The locking dynamics is visualized best by considering individual representative trajectories. When the colliding partners approach each other, the intrinsic angular momentum vector $\mathbf{l}$ (all the dynamical variables below are in reduced units) experiences the locking to the collision axis $\boldsymbol{\rho}$ which causes its reorientation in a space-fixed (SF) frame. Within the AC approximation, the reorientation of $\mathbf{l}$ in the course of the collision is described as a fast precession of $\mathbf{\imath}$ about the slowly rotating collision axis in the region where the momentum is locked to the axis.

One can envisage the following qualitative picture of the evolution of the alignment index $\varsigma(\tau)=\pi_{\phi}(\tau) / \mathrm{l}$ along the trajectory during a collision. During the approach of the partners, $\varsigma(\tau)$ changes first because of the rotation of the collision axis (initial part of the collision, $\mathbf{l}$ is fixed in space, $\mathbf{l}=\mathbf{t}_{i}$ ); then $\varsigma$ becomes approximately constant (middle part of the collision, $\mathbf{l}$ precesses about $\boldsymbol{\rho}$ ). If capture occurs, $\varsigma$ remains the same; in case of reflection (i.e. no capture), $\mathbf{l}$ becomes uncoupled from $\boldsymbol{\rho}$ again, and $\varsigma$ changes again because of the rotation of the collision axis (final part of the collision, $\mathbf{l}$ is fixed in space, $\mathbf{l}=\mathbf{l}_{f}$ ). The net result of the "non-capture" collision then is a certain rotation of the vector $\mathbf{l}$ from its initial state $\mathbf{t}_{i}$ to the final state $\mathbf{l}_{f}$. 
Each trajectory, for a system with fixed chosen values of I and $\imath$, is specified by the initial values $\zeta_{i}$ and $\phi_{i}$. In this Section, we consider the case of pure chargequadrupole interaction (low collision energies) for $s=1$ with the initial values for representative trajectories listed in Table 1. The collision dynamics is represented by plots of three functions, $\rho(\tau), \varsigma(\tau), \nu(\tau)$ in Figures $2-4$. Since the values of $\rho_{i}$ are immaterial (simply $\rho_{i}>>1$ ), the distances $\rho(\tau)$ are normalized to $\rho_{m}$ which is chosen such that the quantities $\rho(\tau) / \rho_{m}$ are fitted into the figure frames.

Figure 2 shows two trajectories (full and dotted lines) with identical values of $\varsigma_{i}$ (marked by the triangle at the left ordinate axis) from the range where AC theory predicts the capture, $\varsigma_{i}>\varsigma_{+}^{\mathrm{AC}, \min }(0)=1 / \sqrt{3}\left(\varsigma_{+}^{\mathrm{AC}, \min }(0)\right.$ is marked by the dotted horizontal line $)$. The values $\phi_{i}$ are slightly different for the two trajectories. The following observations are made:

i) AC theory predicts capture (the AC trajectory is not shown in the Figure). The separation $\rho(\tau)$ decreases and then quite suddenly drops (spiraling to the centre); $\varsigma(\tau)$ remains constant (by assumption, $\varsigma(\tau)=\varsigma_{i}=\omega / 1$ ); its value $\varsigma_{i}$ is marked by the triangle on the left ordinate axis.

ii) The accurate trajectory leading to capture (dotted line). The separation $\rho(\tau)$ decreases, and then quite suddenly drops (spiraling to the centre); $\varsigma(\tau)$ changes regularly for $\tau<-1$, implying the conservation of the direction of the intrinsic momentum in space (no locking). For $\tau>-1, \varsigma(\tau)$ oscillates about a certain mean value $\bar{\zeta}$. The latter is the locked value of the projection, and the amplitude of the oscillations characterizes the strength of the locking. The oscillations of $\varsigma(\tau)$ about $\bar{\zeta}$, which are ignored in the AC approach, are the reflections of the imperfect precession of $\mathbf{j}$ about the collision axis; $\mathbf{a}$ single full oscillation of $\varsigma(\tau)$ corresponds to an increment of the azimuthal angle $\phi$ by $2 \pi$. It is seen that the frequency of the precession during the passage over the potential barrier is much higher than the inverse of the time of the barrier crossing, which is the condition for the applicability of the AC approximation. The number $n$ of precessional revolutions of the intrinsic momentum in the region of the potential barrier along the incoming parts of the trajectories is given in Table 1. We see also that $\bar{\zeta}$ noticeably 
differs from $\varsigma_{i}$, implying that the AC approach cannot be applied to collisions with specific values of $\varsigma_{i}$. Presumably, the AC approach will yield reasonable results on average, i.e. after averaging over $\varsigma_{i}$. The graphs for $v_{e f f}(\tau)$ show what happens with the potential energy: it first increases when the system approaches the barrier and then suddenly drops, following the spiraling to the centre; the maximum value of $v_{\text {eff }}(\tau)$ is below unity.

iii) The accurate trajectory without capture (full line). The incoming part of this trajectory is quite similar to the incoming part of the capture trajectory (see ii). The outgoing part corresponds to a collision with reorientation of the vector $\mathbf{l}$. For this reflected trajectory, the turning point of which corresponds to the maximum of the timedependent potential (equal to unity), the locking gradually becomes weaker, and finally $\varsigma(\tau)$ shows a behavior which is typical for the uncoupled angular momentum. The function $\varsigma(\tau)$ is nearly symmetrical for the incoming and outgoing parts of the trajectory. Note that a small change of the initial angle $\phi_{i}$ noticeably affects the time-dependent potential barrier: it has of a bell-shaped symmetrical form for the reflected trajectory and an asymmetrical form for the capture trajectory. The former feature is related to the almost symmetric shape of the trajectory, while the latter corresponds to a strong acceleration of the relative motion once the barrier is overcome.

For comparison, we show another, quite asymmetrical, function $\varsigma(\tau)$ (dashed line) for a trajectory which starts with $\zeta_{i}$ from the range allowed for capture by the AC approach. Due to the Coriolis interaction, $\varsigma(\tau)$ decreases to such an extent that capture becomes impossible. The locking is quite loose for this trajectory.

Figure 3 refers to the same value of 1 and a smaller value of I, but with the value of $\varsigma_{i}$ from the forbidden region of the AC capture, $\varsigma_{i}<\varsigma_{+}^{\mathrm{AC} \text {,min }}(0)$. Here, the capture turns out to be possible only as a result of the increase in $\varsigma(\tau)$ during the initial part of the collision to such an extent that the barrier can be overcome. The dynamics of the barrier crossing is almost adiabatic with respect to the Coriolis interaction (meaning that the latter can be ignored, $n>>1$ ). Beside the captured and reflected trajectories, which are similar to those in Figure 2, this figure shows also the separatrix (superscript sep), a 
trajectory which comes from infinity and then experiences virtually circular motion around the attractive center (dashed curves). Along this trajectory, in its circular part, $\varsigma(\tau)$ continues to rapidly oscillate about a mean (conserved) value $\bar{\zeta}^{\text {sep }}$ and $\nu_{\text {eff }}^{\text {sep }}(\tau)$ remains approximately constant. The larger number of precessional revolutions in Figure 3 and the smaller amplitude of the oscillations compared to those in Figure 2 are explained by the fact that the impact parameter for the former case is smaller, and, therefore, the locking is tighter.

Figure 4 refers to a higher value of 1 , with the value of $\varsigma_{i}$ from the forbidden region of the AC capture, $\varsigma_{i}<\varsigma_{+}^{\mathrm{AC} \text {,min }}(0)$. Here, the gradual change of $\varsigma(\tau)$ across the whole time interval can be interpreted roughly as the reflection of the rotation of the collision axis with the direction of $\mathbf{j}$ almost fixed in space which is due to higher gyroscopic effects of the rapidly rotating diatom. Indeed, two full oscillations of $\varsigma(\tau)$ in the barrier region reflect a rather weak coupling of $\mathbf{j}$ to $\mathbf{R}$. Note also that in this case the profile of the barrier along the trajectories is of a complicated shape.

\section{Capture cross sections}

Each trajectory for the Hamiltonian in Eqs (6) and (7) defines a capture probability (zero or one) and the set of trajectories, differing in $\phi_{i}$, define the average capture probability $P_{s}\left(\xi_{i}, \mathrm{I}, 1, \lambda\right)$. The latter quantity determines the alignment-specific capture cross section

$\sigma_{s}\left(\varsigma_{i}, 1, \lambda\right)=\pi \int_{\varsigma^{i}}^{\infty} P_{s}\left(\zeta_{i}, \mathrm{I}, \mathrm{l}, \lambda\right) \mathrm{I} d \mathrm{I}$

where $\varsigma_{i}=\cos \beta_{i}$. The AC counterpart of $\sigma_{s}\left(\varsigma_{i}, 1, \lambda\right), \sigma_{s}^{\mathrm{AC}}\left(\varsigma_{i}, \lambda\right)$, that corresponds to the PRAC potential in Eq (8), is

$$
\sigma_{s}^{\mathrm{AC}}\left(\varsigma_{i}, \lambda\right)=\pi \int_{0}^{\infty} P_{s}^{A C}\left(\varsigma_{i}, \mathrm{I}, 1, \lambda\right) \mathrm{I} d \mathrm{I}
$$


where $P_{s}^{A C}\left(\zeta_{i}, \mathrm{I}, 1, \lambda\right)=\Theta\left(1-v_{\mathrm{eff}, s}^{\mathrm{AC}, *}\left(\varsigma^{\mathrm{AC}}, \mathrm{I}, \lambda\right)\right)$ and $\zeta_{i} \equiv \varsigma^{A C}$. Finally, the $\mathrm{FW}$ version of $\sigma_{s}\left(\zeta_{i}, 1, \lambda\right), \sigma_{s}^{\mathrm{FW}}\left(\zeta_{i}, \lambda\right)$, has the form

$$
\sigma_{s}^{\mathrm{FW}}\left(\varsigma_{i}, \lambda\right)=\frac{1}{4 \pi} \int P_{s}^{\mathrm{FW}}\left(\zeta_{i}, \varphi_{i}, \pi_{\theta i}, \varpi, \lambda\right) d \pi_{\theta i} d \varpi d \varphi_{i}
$$

where $P_{s}^{\mathrm{FW}}$ is the capture probability (zero or one), $\varsigma_{i} \equiv \varsigma_{i}^{\mathrm{FW}}=\cos \theta_{i}$, and $\pi_{\theta i}, \theta_{i}, \varphi_{i}$ are the initial values of dynamical variables that enter into the Hamiltonian in Eq (9). Note that neither AC nor FW cross sections depend on 1 . We also mention that the high- $\lambda$ limit of all three cross sections is the scaled Langevin cross section $\sigma^{\mathrm{L}}=\pi \sqrt{\lambda}$.

It was argued in [25] that $\sigma_{s}\left(\zeta_{i}, 1, \lambda\right)$ is expected to be bracketed between $\left.\sigma_{s}^{\mathrm{AC}}\left(\varsigma^{\mathrm{AC}}, \lambda\right)\right|_{\varsigma^{\mathrm{AC}}=\varsigma_{i}}($ for $1<<1)$ and $\left.\sigma_{s}^{\mathrm{FW}}\left(\zeta^{\mathrm{FW}}, \lambda\right)\right|_{\varsigma^{\mathrm{FW}}=\varsigma_{i}}$ (for $\left.1>>1\right)$, though the relation between the two latter quantities could not be predicted a priory. Indeed, as was found earlier [25], the plots $\left.\sigma_{s}^{\mathrm{AC}}\left(\varsigma_{i}, \lambda\right)\right|_{\lambda=0}$ and $\left.\sigma_{s}^{\mathrm{FW}}\left(\varsigma_{i}, \lambda\right)\right|_{\lambda=0}$ vs. $\varsigma_{i}$ cross at a certain point, suggesting that all $\left.\sigma_{s}\left(\varsigma_{i}, 1, \lambda\right)\right|_{\lambda=0}$ pass through (or very close to) this point. This conclusion was exemplified by Figures 3 and 4 in ref [25] for $\imath=0.1$ and $1=0.25$. As follows from the present calculations, this feature of the functions $\sigma_{s}\left(\varsigma_{i}, 1, \lambda\right)$ persists even for different values of $\imath$ and $\lambda$. The above given properties suggest that numerically calculated $\sigma_{s}\left(\zeta_{i}, 1, \lambda\right)$ can be approximately represented by an interpolating expression

$$
\sigma_{s}\left(\zeta_{i}, 1, \lambda\right)=f_{s}^{\mathrm{AC}}(1, \lambda) \sigma_{s}^{\mathrm{AC}}\left(\zeta_{i}, \lambda\right)+f_{s}^{\mathrm{FW}}(1, \lambda) \sigma_{s}^{\mathrm{FW}}\left(\zeta_{i}, \lambda\right)
$$

with fitting functions $f_{s}^{\mathrm{AC}}(1, \lambda)$ and $f_{s}^{\mathrm{FW}}(1, \lambda)$ that describe the dependence of $\sigma_{s}\left(\zeta_{i}, 1, \lambda\right)$ on the scaled intrinsic angular momentum 1 . We have found that, within an accuracy of $15 \%$, the cross sections $\sigma_{s}\left(\varsigma_{i}, 1, \lambda\right)$ are reproduced by Eq (13) with 


$$
f_{s}^{\mathrm{AC}}(\imath, \lambda)=\left(\frac{1}{1+h_{s}(\lambda) \imath^{g_{s}}}\right) ; \quad f_{s}^{\mathrm{FW}}(\imath, \lambda)=\left(\frac{h_{s}(\lambda) 1^{g_{s}}}{1+h_{s}(\lambda) \imath^{g_{s}}}\right)
$$

and

$$
g_{+1}=1.10, g_{-1}=1.35, \quad h_{+}(\lambda)=2+12 \sqrt{\lambda}, \quad h_{-}(\lambda)=14.5 \cdot(1+\lambda)
$$

The essence of the expression in Eq (13) is schematically represented in the set of Figures $5-11$, where the shaded regions cover graphs of $\sigma_{s}\left(\varsigma_{i}, 1, \lambda\right)$ with different values of 1 . Among other things, these figures illustrate how the effect of the initial alignment decreases with increasing $\lambda$, (i.e. with a decrease in the relative anisotropy of interaction).

The capture cross sections for unpolarized diatoms, $\sigma_{s}(l, \lambda), \sigma_{s}^{\mathrm{AC}}(\lambda), \sigma_{s}^{\mathrm{FW}}(\lambda)$, are obtained from $\sigma_{s}\left(\varsigma_{i}, 1, \lambda\right), \sigma_{s}^{\mathrm{AC}}\left(\zeta_{i}, \lambda\right)$ and $\sigma_{s}^{\mathrm{FW}}\left(\zeta_{i}, \lambda\right)$ by averaging over $\varsigma_{i}$ within the range $-1 / 2 \leq \varsigma_{i} \leq 1 / 2$. It turns out that $\sigma_{s}^{\mathrm{AC}}(\lambda)$ and $\sigma_{s}^{\mathrm{FW}}(\lambda)$ are close to each other, and $\sigma_{s}(1, \lambda)$ are bracketed between them in a narrow interval, see Figure 12.

\section{Conclusion}

The collision of molecules in its incoming part is accompanied by a reorientation of the intrinsic angular momenta of the partners from a direction fixed in space to the collision axis (locking). If the interparticle interaction is steep enough compared to the centrifugal interaction, the locking occurs across a narrow interval of interfragment distances. This allows one to separate the reorientation from other inelastic events which can be considered to occur with a conserved projection $J_{R}$ of the total angular momentum $\mathbf{J}$ onto the collision axis $\mathbf{R}$. Such a simplification of the collision dynamics disappears for gradual interactions and the question arises about the effects of a lack of the conservation of $J_{R}$ on the inelastic dynamics.

This article addresses the discussed question by way of examples for the low-energy capture of a charged particle (an ion) by a rotating quadrupolar homonuclear 
diatomic molecule. The energy range considered is limited by the conditions of applicability of the perturbed-rotor approximation (upper boundary), which also guaranties the adiabatic behaviour with respect to transitions between rotational states of the diatom [27], and of the applicability of a classical description of the relative motion (lower boundary). The ion-molecule interaction is restricted to the long-range potential that includes anisotropic charge-quadrupole and isotropic charge-induced dipole terms. The former, featuring $R^{-3}$ dependence, is the most slowly-varying interaction among the inverse-power dependences of the $R^{-n}$ type for which a locking distance and range can be defined, and, therefore, is a most interesting optimal candidate for studying the effect of non-conservation of $J_{R}$.The classical Hamiltonian derived in the perturbed-rotor approximation describes the relative radial motion of the partners and the reorientation of the intrinsic angular momentum (its magnitude $j$ being conserved) of the diatom with respect to the BF frame (Section 2). The capture dynamics is visualized by the analysis of trajectories that demonstrate the locking and the passage/reflection via/from centrifugal barriers for representative initial conditions of the initial rotational polarization of the diatom (Section 3). The dependence of the capture cross section on the initial rotational alignment of the diatom with respective to the relative velocity vector is found to be noticeably different from that adopted in the standard adiabatic channel (AC) treatment of the capture, with minimum deviations from AC predictions for small intrinsic momenta (Section 3). These numerical results are summarized by fitting interpolation equations (4.5) that analytically represent the scaled alignment specific cross sections for different scaled values of intrinsic angular momenta and different anisotropies of the interaction. The results are relevant for the calculation of $j$-specific cross sections and rate coefficients for formation of the complexes in collisions of ions with rotationallypolarized homonuclear diatomic molecules. For the capture of unpolarized molecules, one still can use the AC approach for calculating the low-energy capture cross sections, provided that inaccuracies in the capture cross sections of about 5-10\% can be tolerated, although the formal condition of the applicability of the AC approach (conservation of the projection of the intrinsic angular momentum onto the collision axis) is not fulfilled. 


\section{Acknowledgement}

We thank R. N. Zare for his seminal contribution to this field [26] and his continuous interest in our work. 
References

[1] C.T. Rettner and R.N. Zare, J. Chem. Phys. 77, 2418 (1982).

[2] I.V. Hertel, Adv. Chem. Phys. 45, 341 (1980).

[3] E.I. Dashevskaya and N.A. Mokhova, Chem. Phys. Lett. 20, 454 (1973).

[4] I.V. Hertel, H. Schmidt, A. Baering and E. Meyer, Rep. Prog. Phys. 48, 375 (1985).

[5] E.E.B. Campbell, H. Schmidt and I.V. Hertel, Adv. Chem. Phys. 75, 37 (1988).

[6] N. Andersen and K. Bartschat, Polarization, Alignment, and Orientation in Atomic Collisions (Springer, New York, 2001).

[7] B. Pouilly and M.H. Alexander, Chem. Phys. 145, 191 (1990).

[8] A. Berengolts, E.I. Dashevskaya, E.E. Nikitin and J. Troe, Chem. Phys. 195, 283 (1995).

[9] E.E. Nikitin and S.Ya. Umanskii, Theory of Slow Atomic Collisions (Springer, Berlin, 1984).

[10] E.E. Nikitin and R.N. Zare, Mol. Phys. 82, 85 (1994).

[11] V. Aquilanti, S. Cavalli and G. Grossi, Z. Phys. D 36, 215 (1996).

[12] V.V. Kuznetzov and O.S. Vasyutinskii, J. Chem. Phys. 128, 194314 (2008).

[13] V.V. Kuznetzov, S. Sternin and O.S. Vasyutinskii, J. Chem. Phys. 130, 134312 (2009).

[14] D.A. Case and R.D. Herschbach, Mol. Phys. 30, 1537 (1975).

[15] D.A. Case and R.D. Herschbach, J. Chem. Phys. 64, 4212 (1976).

[16] E.I. Dashevskaya, E.E.Nikitin and J. Troe, J. Chem. Phys. 97, 3318 (1992).

[17] M. Quack and J. Troe, Ber. Bunsenges. Phys. Chem. 78, 240 (1974); 79, 170, 469 (1975).

[18] K. Sakimoto and K. Takayanagi, J. Phys. Soc. Japan 48, 2076 (1980).

[19] D.R. Bates, Proc. Roy. Soc. London A 384, 289 (1982).

[20] D.C. Clary, Mol. Phys. 54, 605 (1985).

[21] J. Troe, in State selected and State-to-State Ion-Molecule Reaction Dynamics, Part 2: Theory, edited by M. Baer and C.Y. Ng (Wiley, New York), Adv. in Chem. Phys. 82, 485 (1992). 
[22] M. Ramillon and R. McCarroll, J. Chem. Phys. 101, 8697 (1994).

[23] A.I. Maergoiz, E.E. Nikitin, J. Troe and V.G. Ushakov, J. Chem. Phys. 105, 6270 (1996).

[24] E.I. Dashevskaya, I. Litvin, E.E. Nikitin and J. Troe, J. Chem. Phys. 122, 184311 (2005).

[25] E.I. Dashevskaya, I. Litvin and E.E. Nikitin, J. Phys. Chem. A 110, 2786 (2006).

[26] R.N. Zare, Angular Momentum (Wiley, New York, 1988).

[27] E.E. Nikitin and J. Troe, Phys. Chem. Chem. Phys. 7, 1 (2005). 
Table 1. Initial conditions for the two trajectories shown in Figures 2-4: total angular momentum I, intrinsic angular momentum 1 , initial alignment index $\varsigma_{i}$, initial angle $\phi_{i}$, scaling distances $\rho_{m}$, number $n$ of precessional revolutions of the intrinsic momentum in the region of the potential barrier along incoming parts of the trajectories, and the trajectory types.

\begin{tabular}{|c|c|c|c|c|c|c|l|}
\hline Figure & $\mathrm{I}$ & $\mathrm{l}$ & $\varsigma_{i}$ & $\rho_{\mathrm{m}}$ & $\phi_{i} / \pi$ & $n$ & \multicolumn{1}{|c|}{ Trajectory type } \\
\hline 2 & 1.0 & 0.1 & 0.80 & 5.29 & 0.470 & $\approx 9$ & Capture, with AC open \\
& & & & & 0.485 & & Reflection \\
\hline 3 & 0.55 & 0.1 & 0.52 & 3.17 & 0.045 & 28.0 & Capture, with AC closed \\
& & & & & 0.050 & & Reflection \\
\hline 4 & 1.3 & 1.0 & 0.1 & 11.5 & -0.09 & \multirow{2}{*}{$\approx 1$} & Capture, with AC closed \\
& & & & & -0.08 & & Reflection \\
\hline
\end{tabular}


Figure Captions

Figure 1. Minimum and maximal values of the $A C$ alignment indices $\varsigma_{-}^{A C, \text { max }}$ and $\varsigma_{+}^{A C, \min }$ vs. the relative strength of the isotropic/anisotropic interaction parameter $\lambda$.

Figure 2. Collision dynamics for $s=+1$ along two trajectories for low value of 1 $(l=0.1)$ with initial conditions from the allowed AC range (initial conditions are listed in Table 1). Shown are the scaled interfragment distance $\rho(\tau) / \rho_{m}$, alignment index $\varsigma(\tau)$, reduced effective potential energy $v_{\text {eff }}(\tau)$. The full lines refer to the reflected trajectory, dots to the captured trajectory. The symbols at the ordinate axis mark asymptotic (initial for captured and reflected trajectories, final for reflected trajectory) values of $\varsigma$ and the dotted horizontal line divides open and closed capture states in the AC approximation. Dashed line illustrates the evolution of $\varsigma(\tau)$ along a reflected trajectory from an initial value in the allowed $\mathrm{AC}$ region to the final value in the forbidden $\mathrm{AC}$ region.

Figure 3. The same as in Figure 2 but with the initial conditions from the forbidden AC range (see Table 1). Also shown are the similar attributes for the separatrix (dashed lines): interfragment distance $\rho^{\text {sep }}(\tau) / \rho_{m}$, potential energy $v_{\text {eff }}^{\text {sep }}(\tau)$, and the filled envelope for fast oscillations of $\varsigma^{\text {sep }}(\tau)$.

Figure 4. The same as in Figure 2 but for a higher value of $1(l=1)$ with initial conditions from the forbidden AC range (see Table 1).

Figure 5. Dependence of the reduced alignment-specific cross sections $\sigma_{s}\left(\varsigma_{i}, 1, \lambda\right)$ on the initial alignment index $\zeta_{i}$ for different $\imath$ and $\lambda=0$. The plots of $\sigma_{s}\left(\zeta_{i}, 1, \lambda\right)$ lie in the shaded regions bounded by two curves, $\sigma_{s}^{\mathrm{AC}}\left(\varsigma_{i}, \lambda\right)$ and $\sigma_{s}^{\mathrm{FW}}\left(\varsigma_{i}, \lambda\right)$.

Figure 6. As Figure 5 but for $\lambda=0.1$ 
Figure 7. As Figure 5 but for $\lambda=0.2$

Figure 8 . As Figure 5 but for $\lambda=0.5$

Figure 9. As Figure 5 but for $\lambda=1$

Figure 10. As Figure 5 but for $\lambda=2$

Figure 11. As Figure 5 but for $\lambda=5$

Figure 12. The dependence of the alignment-averaged cross sections $\bar{\sigma}_{s}(1, \lambda)$ on the relative strength of the isotropic/anisotropic interaction parameter $\lambda$. The plots of $\bar{\sigma}_{s}(1, \lambda)$ lie in the shaded regions bounded by two curves, $\bar{\sigma}_{s}^{\mathrm{AC}}(\lambda), \bar{\sigma}_{s}^{\mathrm{FW}}(\lambda)$. 
Figures

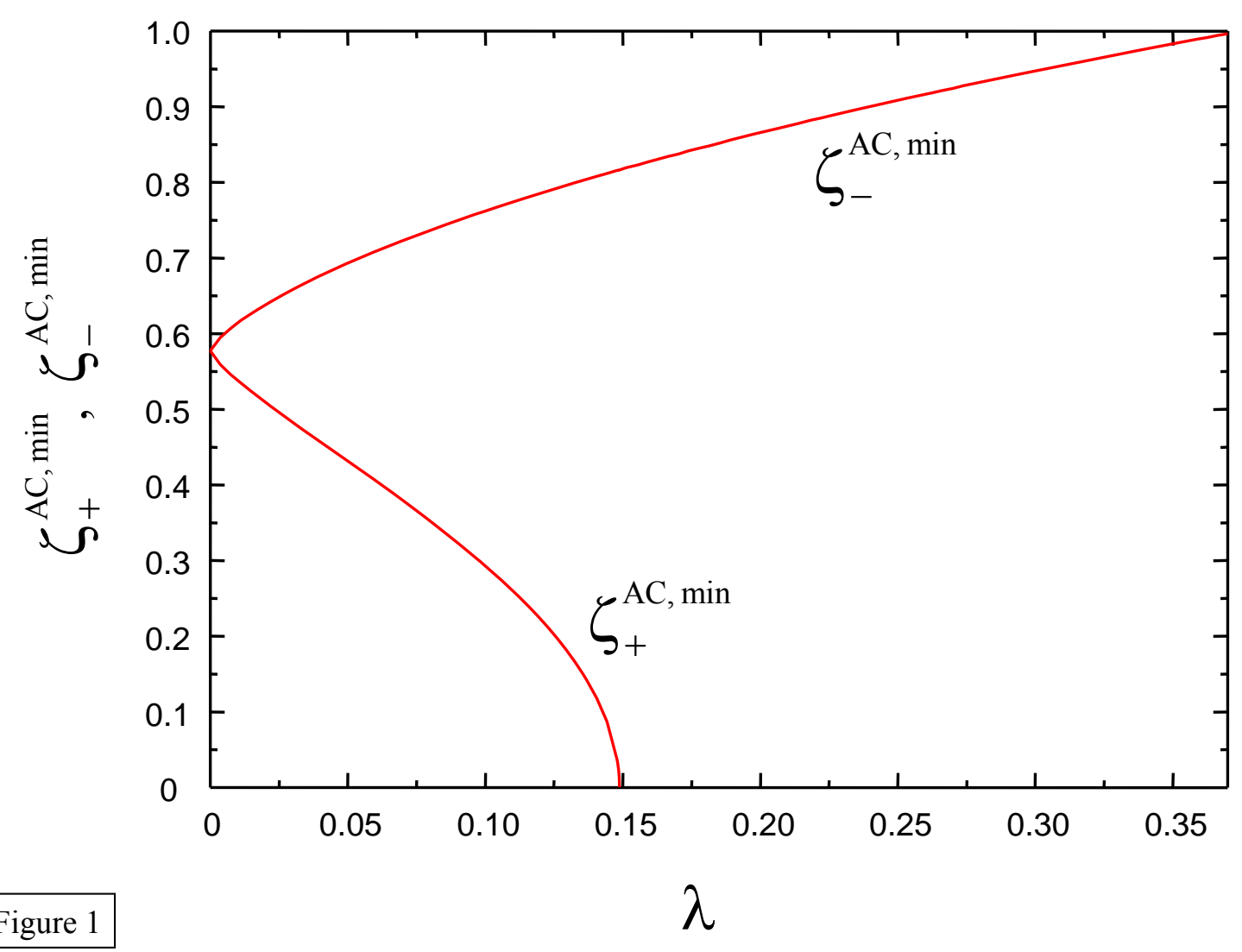

Figure 1. Minimum and maximal values of the AC alignment indices $\varsigma_{-}^{A C, \max }$ and $\varsigma_{+}^{A C, \min }$ vs. the relative strength of the isotropic/anisotropic interaction parameter $\lambda$. 


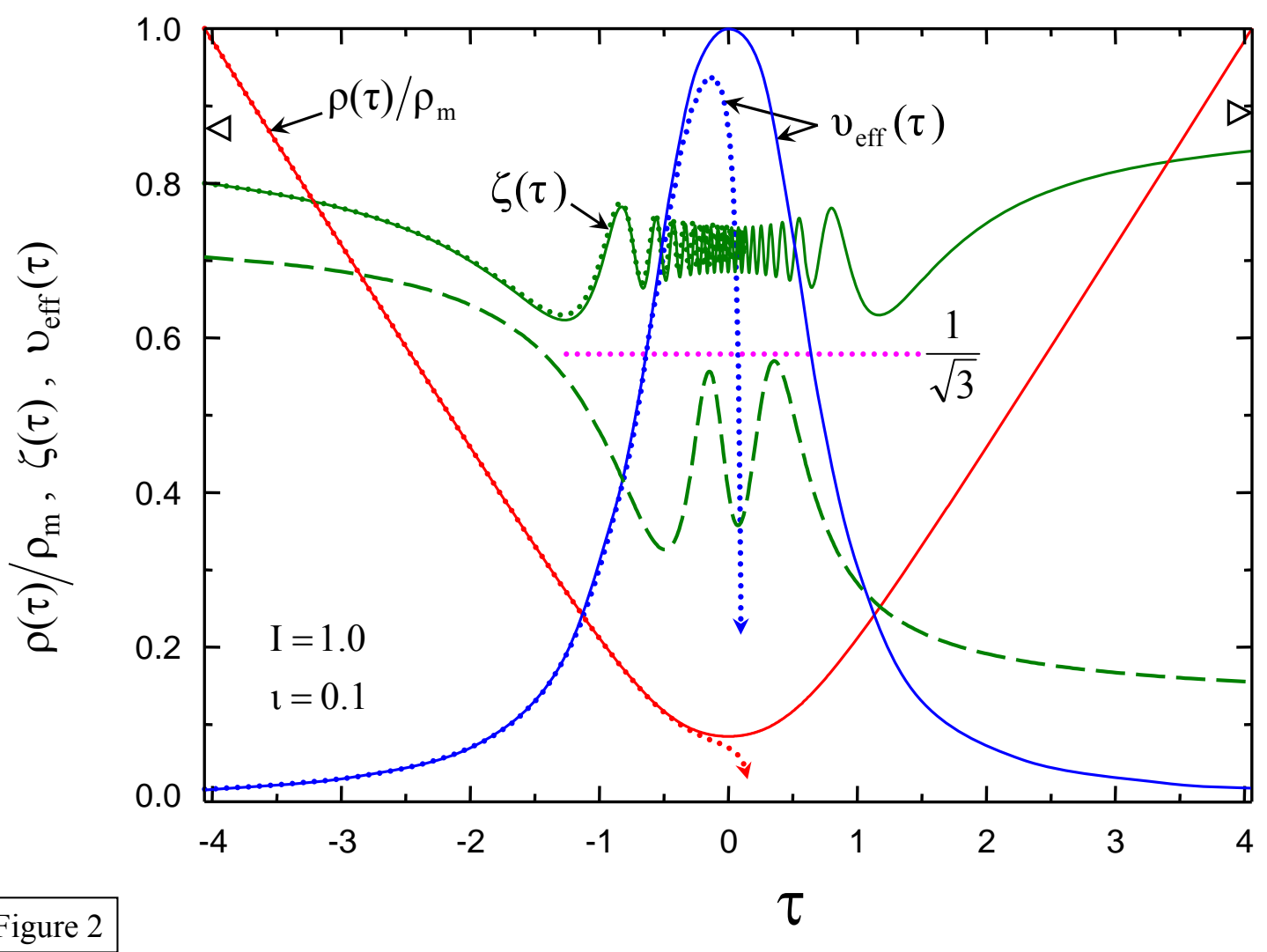

Figure 2. Collision dynamics for $s=+1$ along two trajectories for low value of 1 $(l=0.1)$ with initial conditions from the allowed AC range (initial conditions are listed in Table 1). Shown are the scaled interfragment distance $\rho(\tau) / \rho_{m}$, alignment index $\varsigma(\tau)$, reduced effective potential energy $v_{\text {eff }}(\tau)$. The full lines refer to the reflected trajectory, dots to the captured trajectory. The symbols at the ordinate axis mark asymptotic (initial for captured and reflected trajectories, final for reflected trajectory) values of $\varsigma$ and the dotted horizontal line divides open and closed capture states in the AC approximation. Dashed line illustrates the evolution of $\zeta(\tau)$ along a reflected trajectory from an initial value in the allowed $\mathrm{AC}$ region to the final value in the forbidden $\mathrm{AC}$ region. 


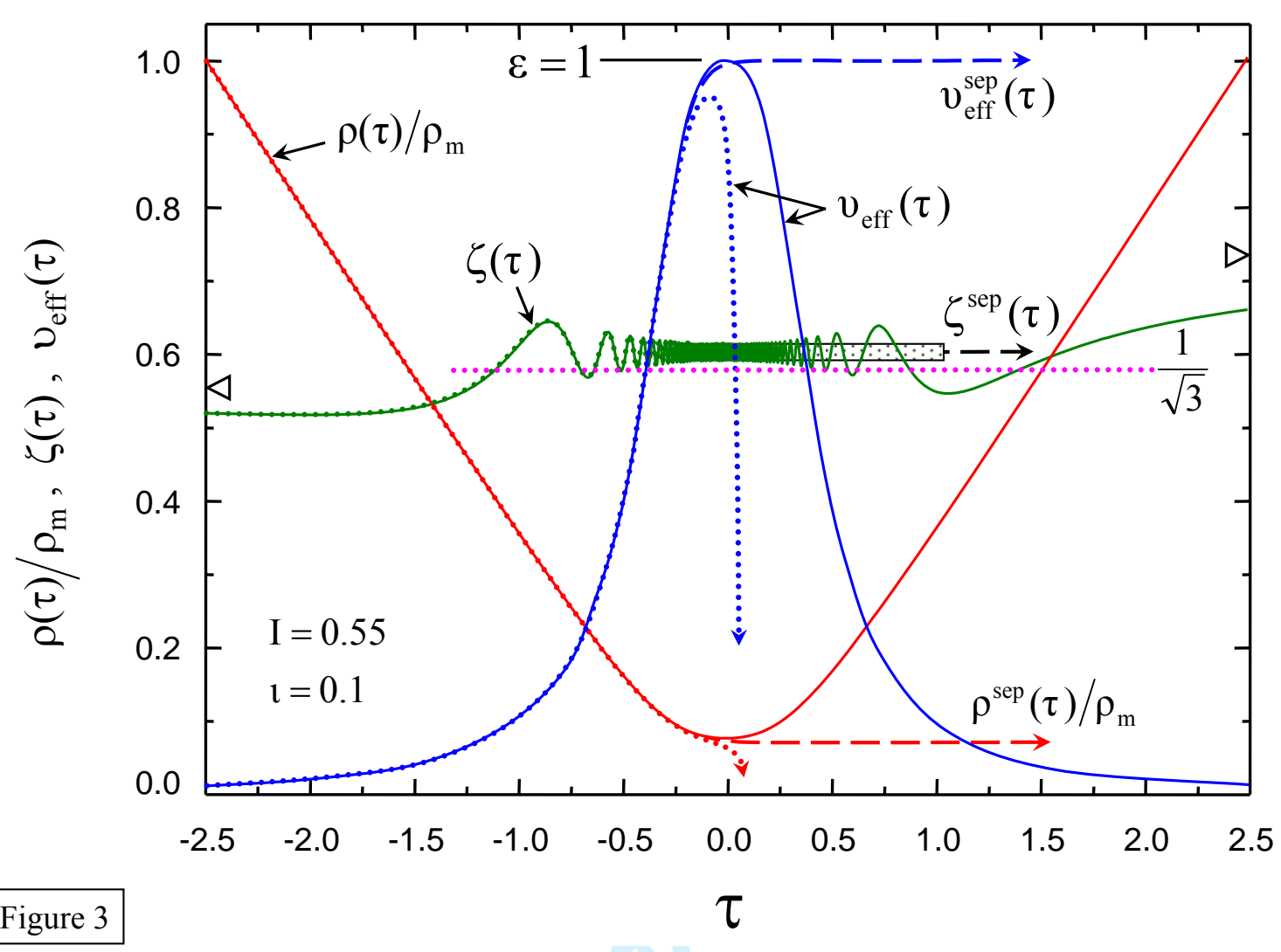

Figure 3. The same as in Figure 2 but with the initial conditions from the forbidden AC range (see Table 1). Also shown are the similar attributes for the separatrix (dashed lines): interfragment distance $\rho^{\mathrm{sep}}(\tau) / \rho_{m}$, potential energy $\cup_{\mathrm{eff}}^{\mathrm{sep}}(\tau)$, and the filled envelope for fast oscillations of $\varsigma^{\mathrm{sep}}(\tau)$. 


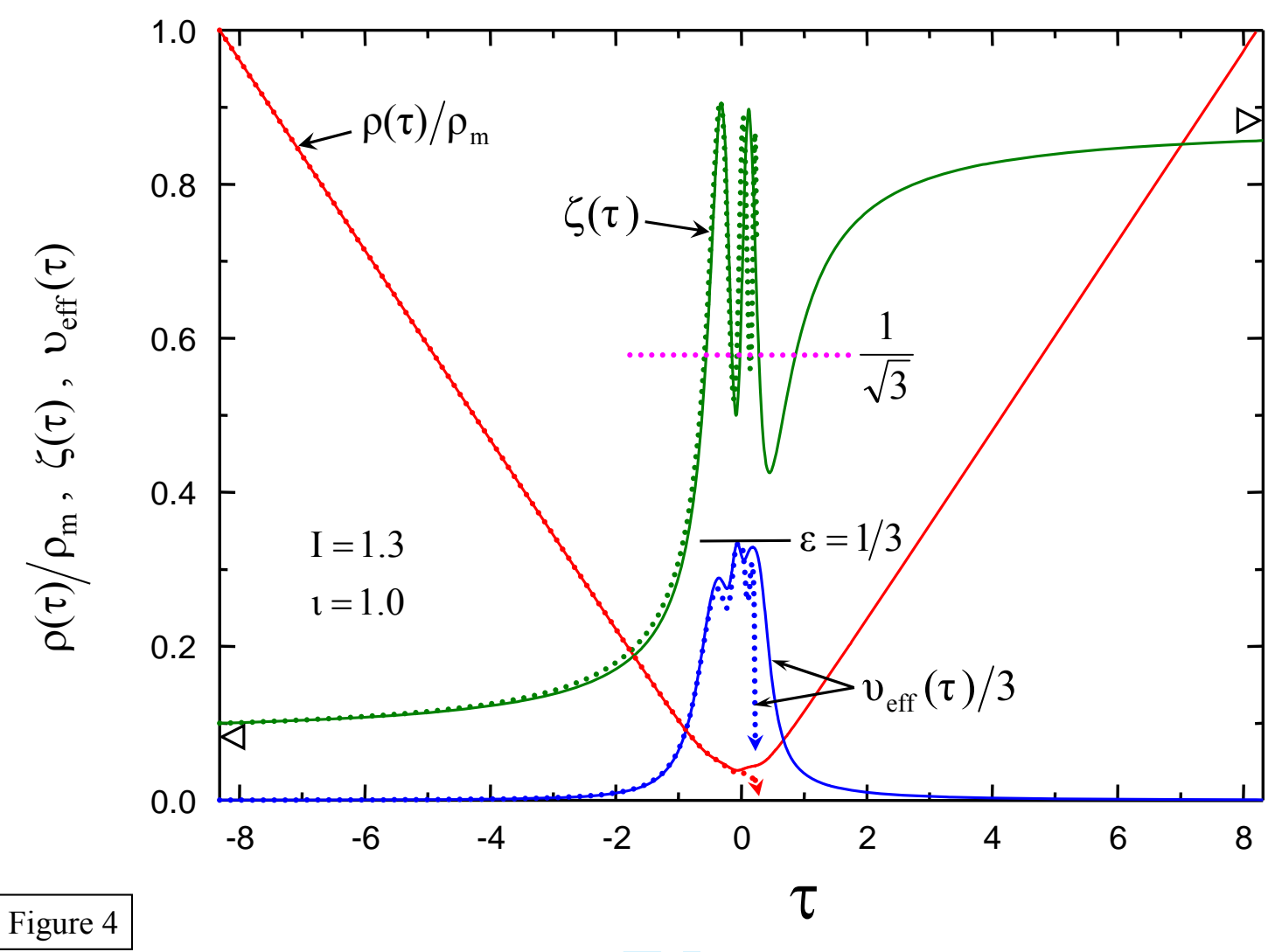

Figure 4. The same as in Figure 2 but for a higher value of $\imath(l=1)$ with initial conditions from the forbidden AC range (see Table 1). 


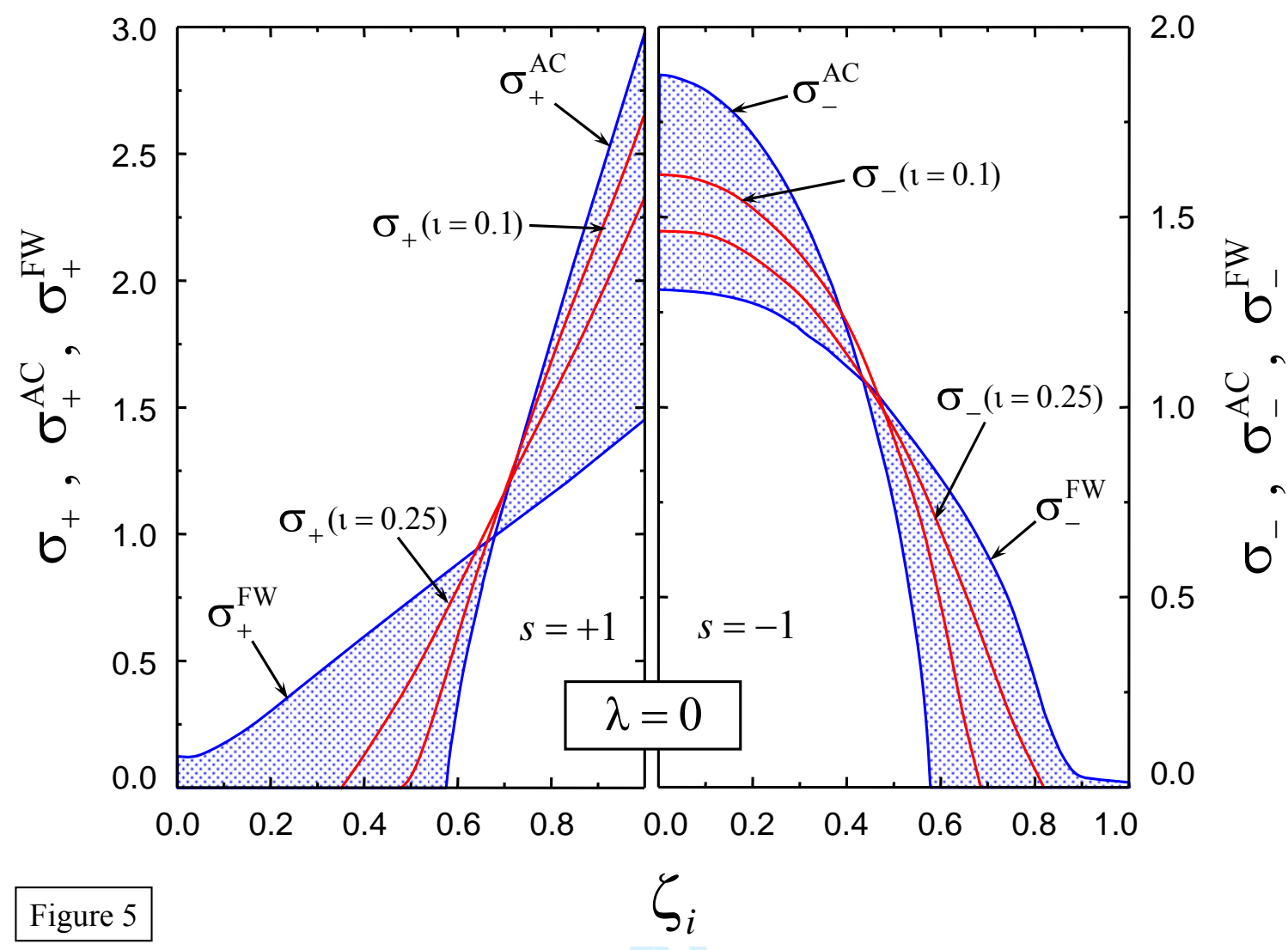

Figure 5. Dependence of the reduced alignment-specific cross sections $\sigma_{s}\left(\varsigma_{i}, 1, \lambda\right)$ on the initial alignment index $\zeta_{i}$ for different $\imath$ and $\lambda=0$. The plots of $\sigma_{s}\left(\zeta_{i}, 1, \lambda\right)$ lie in the shaded regions bounded by two curves, $\sigma_{s}^{\mathrm{AC}}\left(\varsigma_{i}, \lambda\right)$ and $\sigma_{s}^{\mathrm{FW}}\left(\varsigma_{i}, \lambda\right)$. 


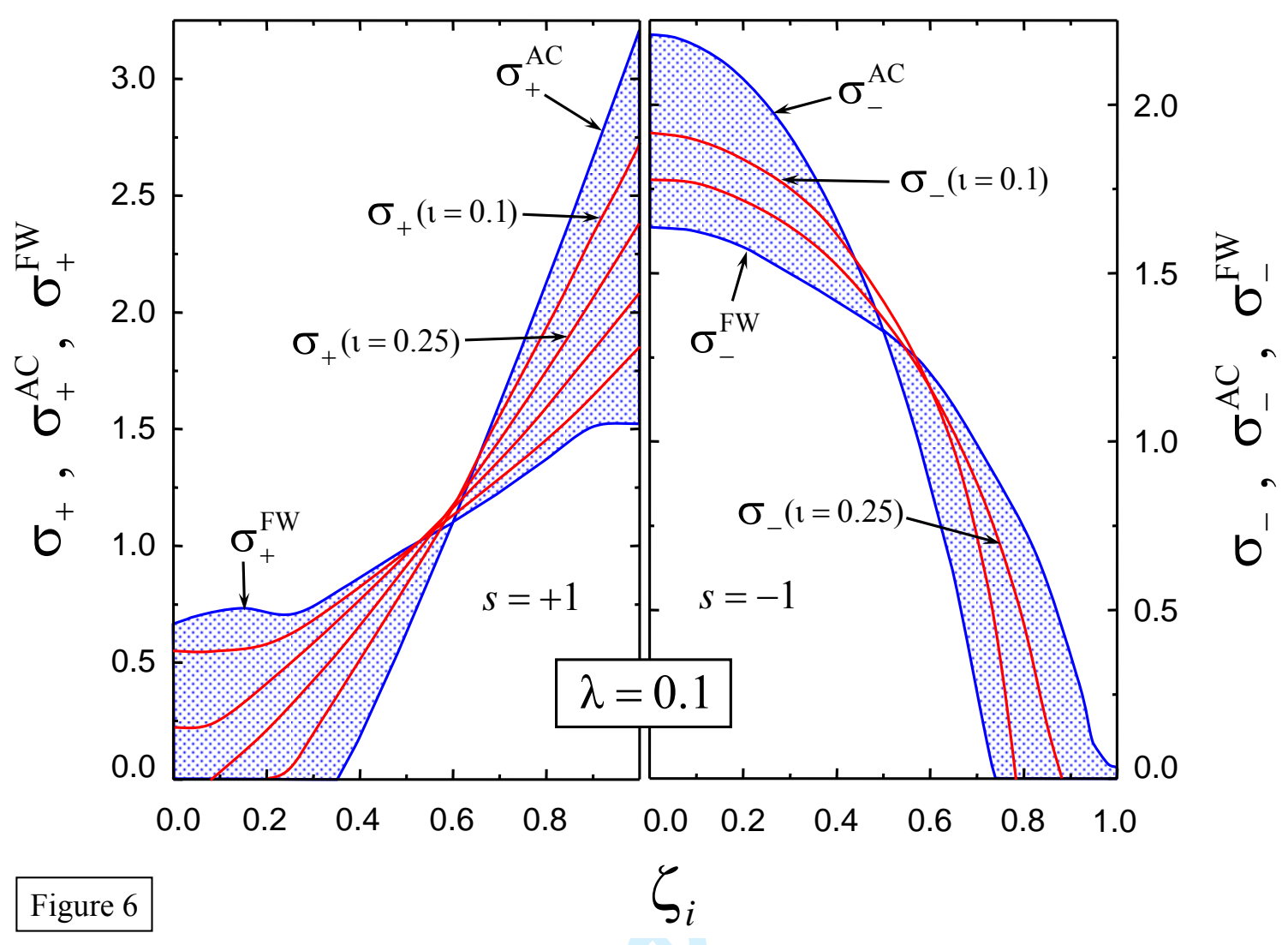

Figure 6. As Figure 5 but for $\lambda=0.1$ 


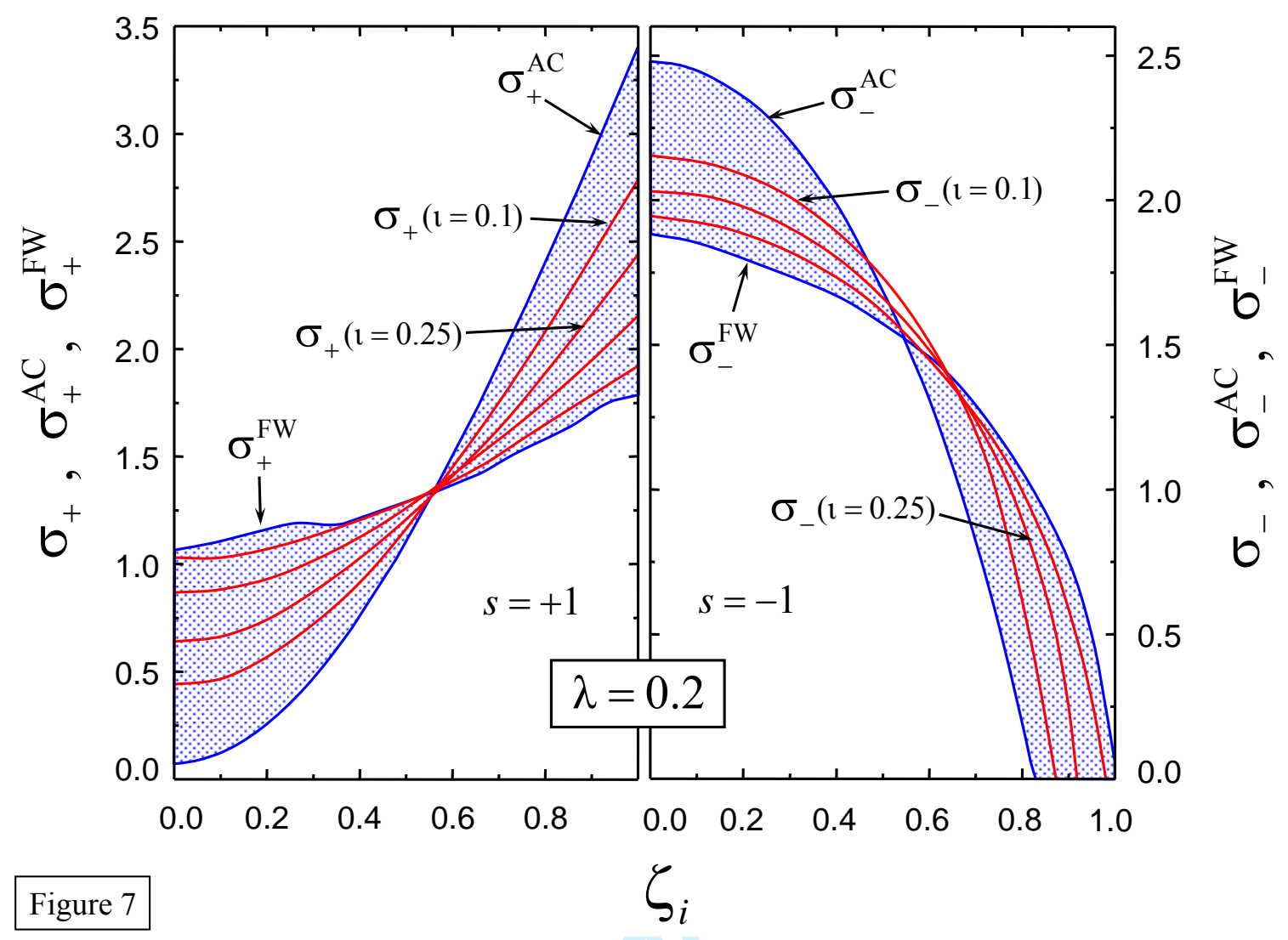

Figure 7. As Figure 5 but for $\lambda=0.2$ 


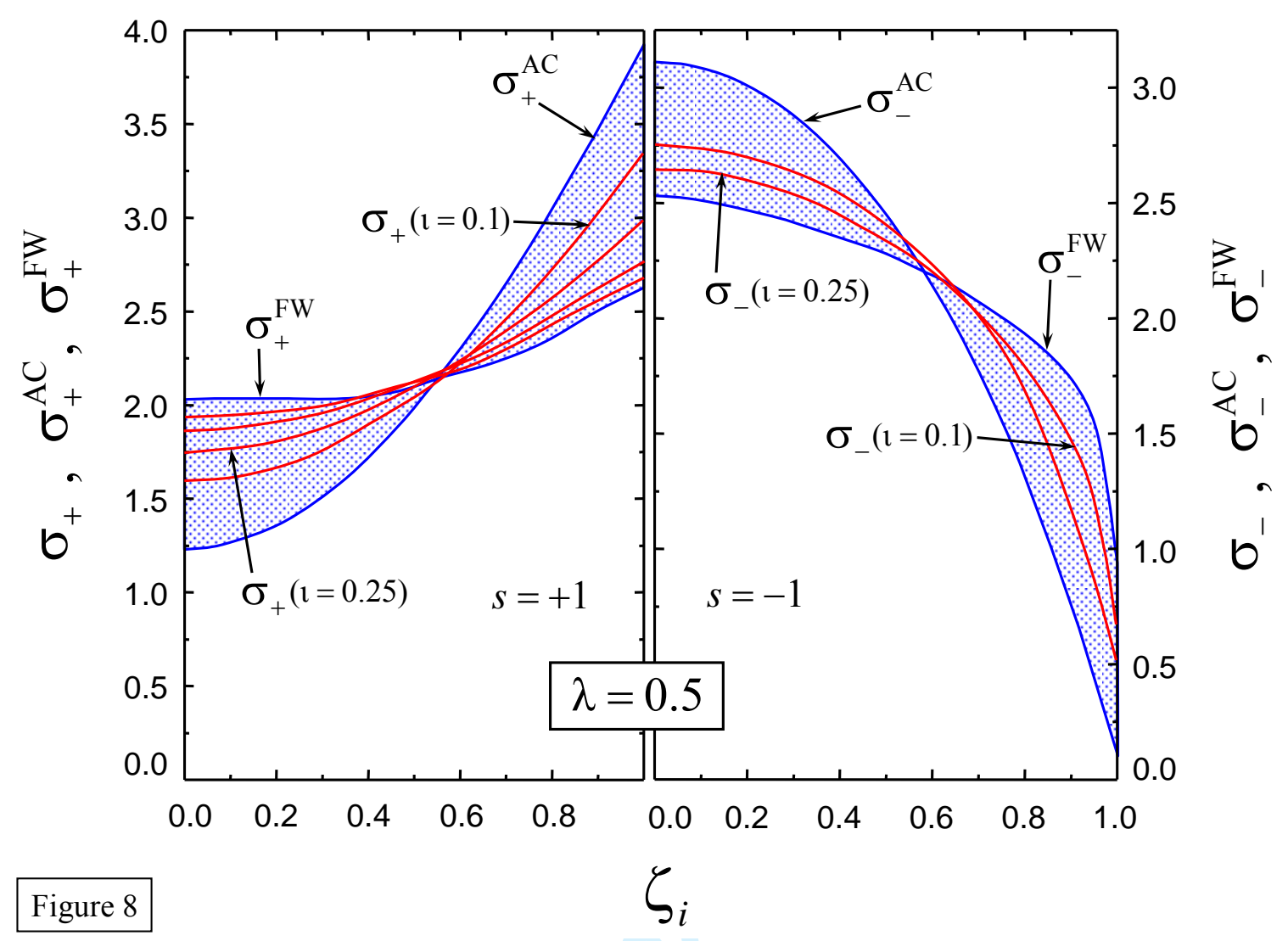

Figure 8 . As Figure 5 but for $\lambda=0.5$ 


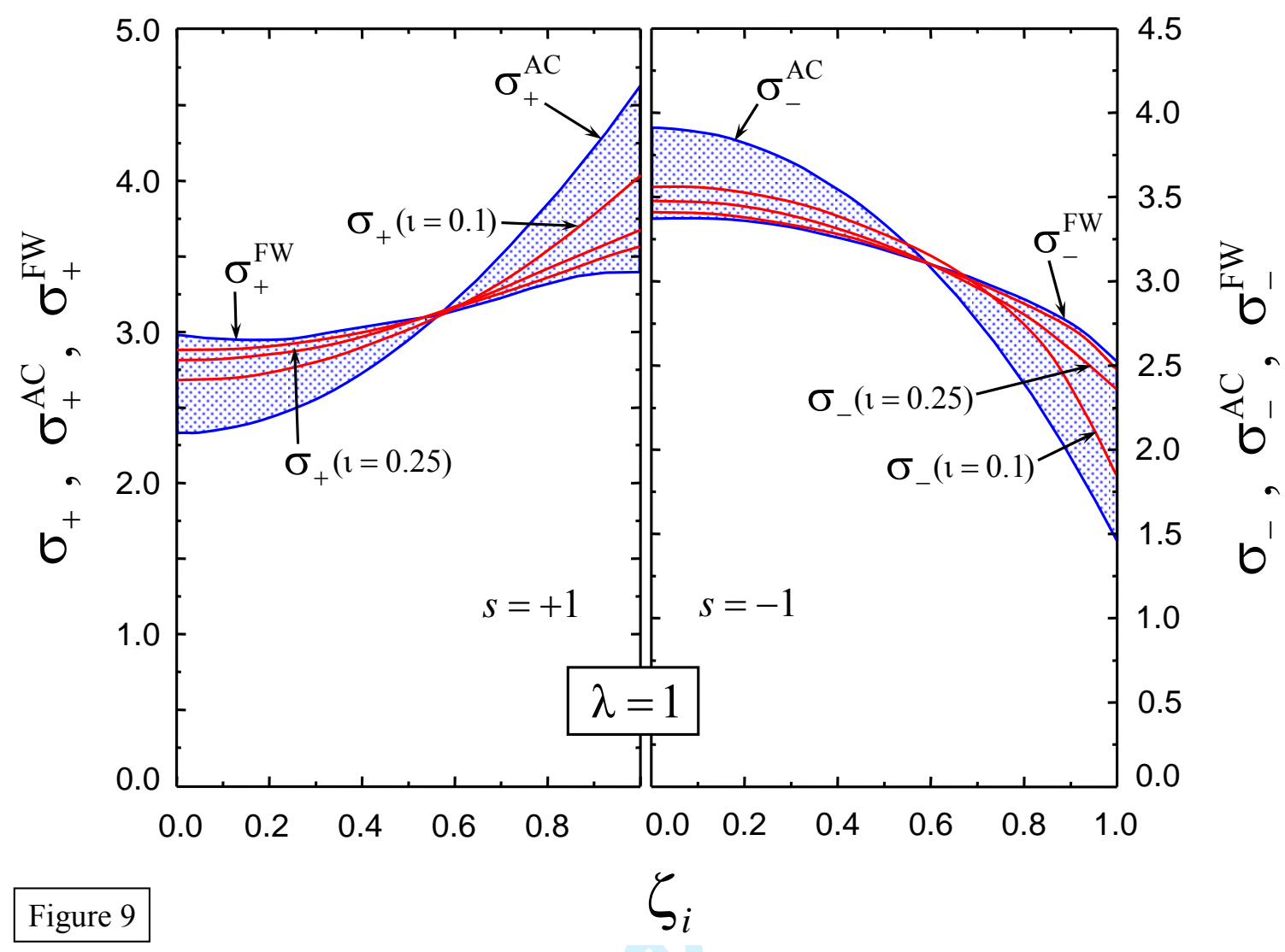

Figure 9. As Figure 5 but for $\lambda=1$ 


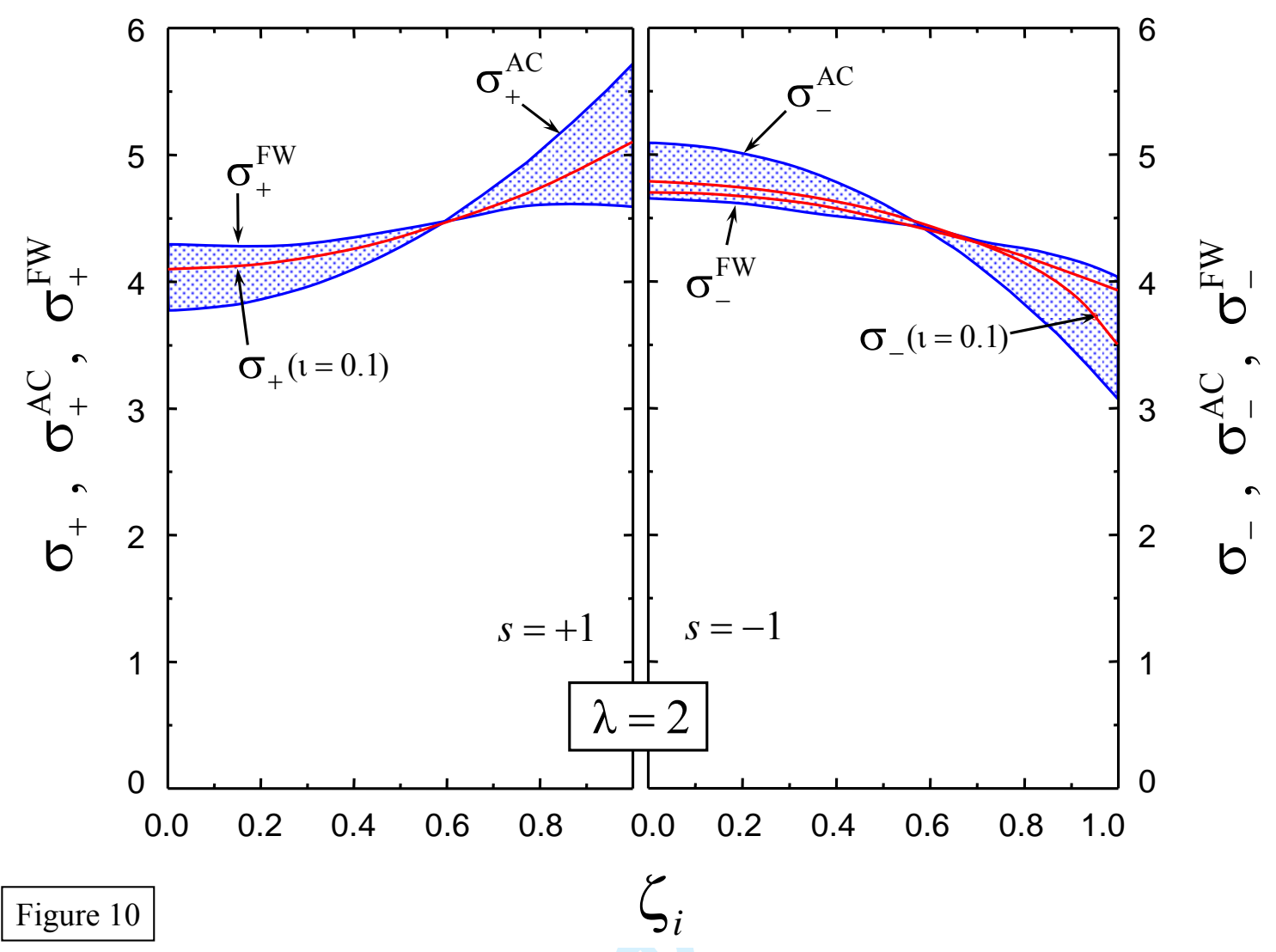

Figure 10. As Figure 5 but for $\lambda=2$ 


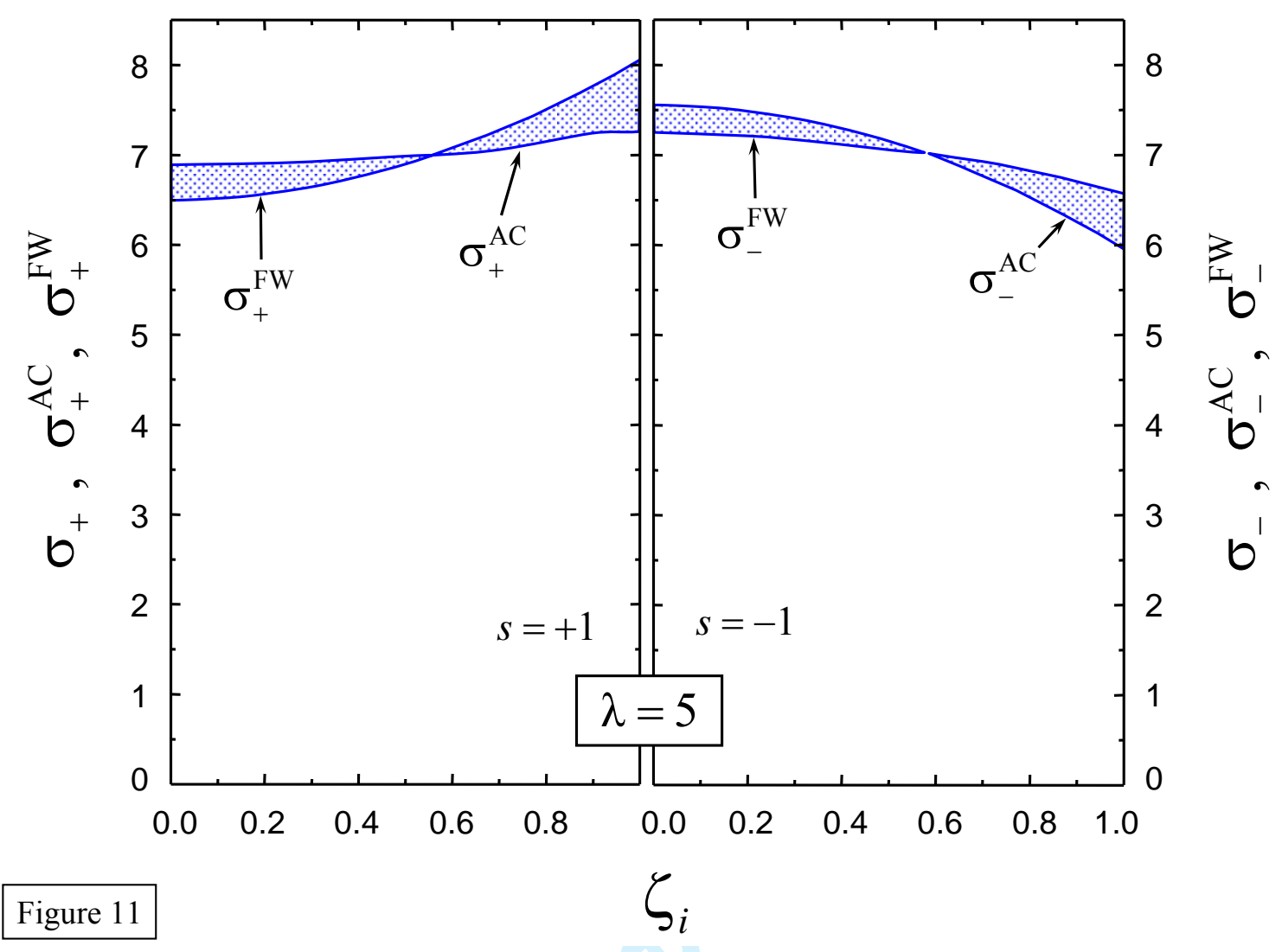

Figure 11. As Figure 5 but for $\lambda=5$ 


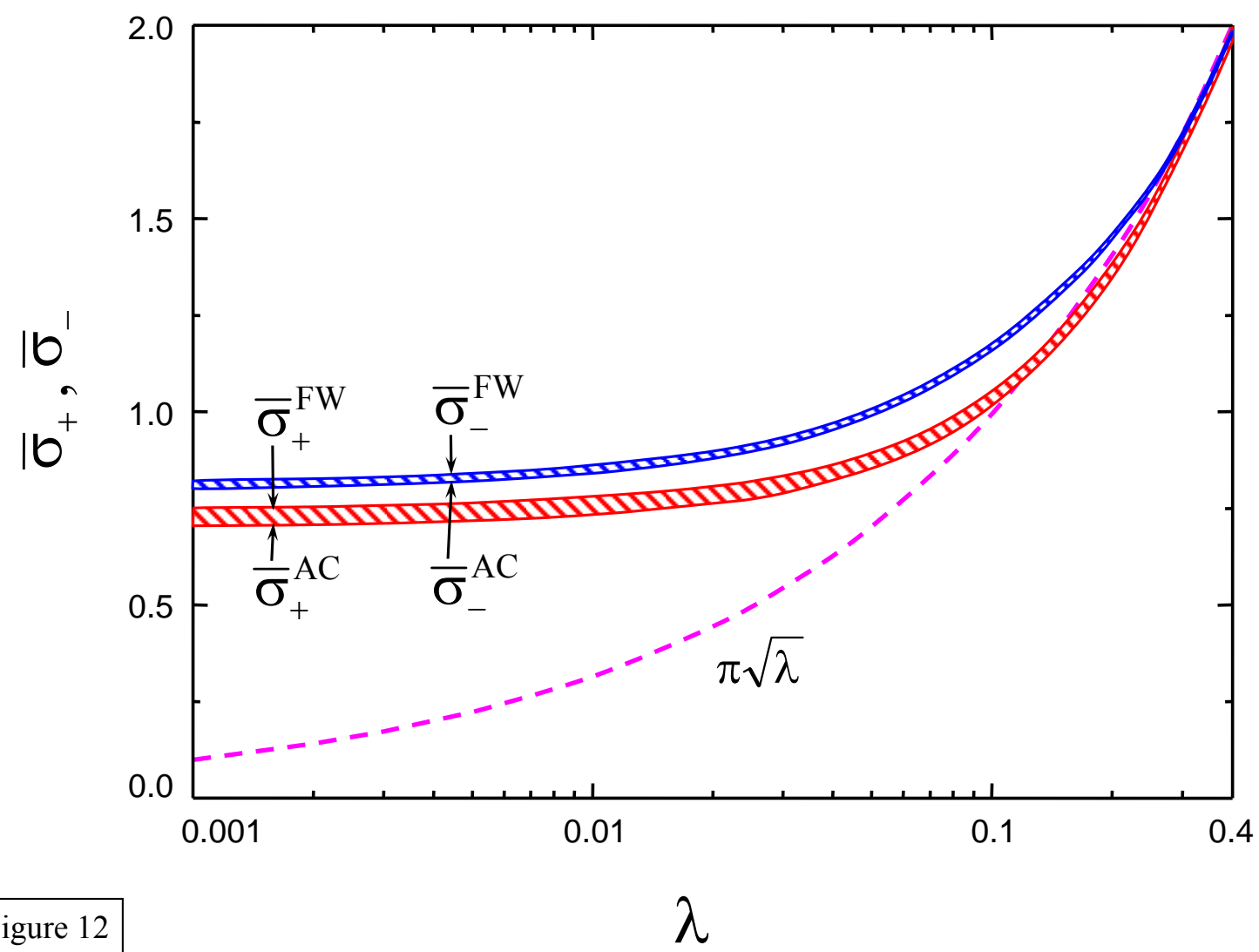

Figure 12. The dependence of the alignment-averaged cross sections $\bar{\sigma}_{s}(1, \lambda)$ on the relative strength of the isotropic/anisotropic interaction parameter $\lambda$. The plots of $\bar{\sigma}_{s}(1, \lambda)$ lie in the shaded regions bounded by two curves, $\bar{\sigma}_{s}^{\mathrm{AC}}(\lambda), \bar{\sigma}_{s}^{\mathrm{FW}}(\lambda)$. 